ECCOMAS

Proceedia

\title{
STOCHASTIC NONLINEAR RESPONSE OF STRUCTURAL SYSTEMS ENDOWED WITH SINGULAR MATRICES SUBJECT TO COMBINED PERIODIC AND STOCHASTIC EXCITATIONS
}

\author{
I.P. Mitseas ${ }^{1,2}$, P. Ni ${ }^{3}$, V.C. Fragkoulis ${ }^{3}$, F. Kong ${ }^{4}$, M. Beer ${ }^{3,5,6}$ and M. Fragiadakis ${ }^{1}$ \\ ${ }^{1}$ School of Civil Engineering, National Technical University of Athens \\ Iroon Polytechneiou 9, Zografou 15780, Athens, Greece \\ e-mail: Imitseas@mail.ntua.gr, Mfrag@mail.ntua.gr \\ ${ }^{2}$ School of Civil Engineering, University of Leeds \\ Leeds LS2 9JT, UK \\ e-mail: I.Mitseas@leeds.ac.uk \\ ${ }^{3}$ Institute for Risk and Reliability, Leibniz University Hannover \\ Callinstr. 34, Hannover 30167, Germany \\ e-mail: peihua.ni@irz.uni-hannover.de, fragkoulis@irz.uni-hannover.de, beer@irz.uni-hannover.de \\ ${ }^{4}$ School of Civil Engineering and Architecture, Wuhan University of Technology \\ 122 Luoshi Road, Wuhan 430070, China \\ e-mail: kongfan@whut.edu.cn \\ ${ }^{5}$ Institute of Risk and Uncertainty, University of Liverpool \\ Peach Street, Liverpool L69 7ZF, UK
${ }^{6}$ International Joint Research Center for Engineering Reliability and Stochastic Mechanics, Tongji University Shanghai 200092, China

\begin{abstract}
A new technique is proposed for determining the response of multi-degree-of-freedom nonlinear complex multi-body systems with singular parameter matrices subject to combined periodic and stochastic loads. The singular parameter matrices are due to adopting redundant coordinates for modeling the system governing equations of motion. Considering that the system response has a periodic as well as a stochastic component, a harmonic balance methodbased scheme is used for treating the deterministic component, followed by the application of the generalized statistical linearization method, in conjunction with an averaging treatment to account for the stochastic component. The validity of the proposed technique is demonstrated by pertinent numerical examples.
\end{abstract}

Keywords: Singular Matrix, Combined Periodic and Stochastic Excitations, Moore-Penrose Inverse Matrix, Nonlinear System, Statistical Linearization. 


\section{INTRODUCTION}

When the problem of formulating the governing equations of motion of multi-degree-offreedom (MDOF) dynamical systems is considered, the minimum number of independent coordinates is used. Nevertheless, for several classes of complex multi-body systems and also systems subject to constraints [1,2], a redundant coordinates modeling is often connected with solution treatments of reduced computational cost $[3,4,5]$. However, this yields singular matrices in the system governing equations of motion. Therefore, several techniques have been developed recently for conducting stochastic response analyses pertaining to systems exhibiting singular parameter matrices; see, indicatively, $[6,7,8,9,10,11]$. This is achieved by resorting to the theory of generalized matrix inverses.

In this paper, a Moore-Penrose (M-P) generalized matrix inverses framework is adopted for determining the response of MDOF nonlinear systems with singular matrices subject to combined periodic and stochastic excitations. To this end, the harmonic balance method is combined with the statistical linearization methodology for systems with singular matrices $[12,13]$. Specifically, since the system excitation consists of a periodic and a stochastic component, the corresponding response also has a periodic and a stochastic part. Therefore, a solution treatment based on the harmonic balance method is used for the former, whereas the latter is treated by resorting to the generalized statistical linearization method. Combining the two methods results in the formulation of a coupled system of algebraic equations, which is solved iteratively by invoking the generalized matrix inverse theory [14]. Overall, the proposed technique consists a generalization of the framework developed in [15] to account for systems with singular matrices. Its validity is demonstrated by pertinent numerical examples.

\section{MATHEMATICAL FORMULATION}

The governing equations of motion of an $l$-DOF nonlinear system are given by

$$
\mathbf{M}_{\mathbf{x}} \ddot{\mathbf{x}}+\mathbf{C}_{\mathbf{x}} \dot{\mathbf{x}}+\mathbf{K}_{\mathbf{x}} \mathbf{x}+\Phi_{\mathbf{x}}(\mathbf{x}, \dot{\mathbf{x}}, \ddot{\mathbf{x}})=\mathbf{f}_{d, \mathbf{x}}(t)+\mathbf{Q}_{\mathbf{x}}(t),
$$

where $\mathbf{x}$ denotes an $l$ dependent coordinates vector, $\mathbf{M}_{\mathbf{x}}, \mathbf{C}_{\mathbf{x}}$ and $\mathbf{K}_{\mathbf{x}}$ are, respectively, the $l \times l$ mass, damping and stiffness matrices, and $\Phi_{\mathbf{x}}(\mathbf{x}, \dot{\mathbf{x}}, \ddot{\mathbf{x}})$ represents the $l$ vector of the nonlinearities, which are of the polynomial kind for simplicity. Further, $\mathbf{f}_{d, \mathbf{x}}(t)$ represents the periodic component of the excitation, whereas $\mathbf{Q}_{\mathbf{x}}(t)$ is a zero-mean Gaussian stochastic excitation. Considering that the system of Eq. (1) is subject to a number of constraint equations of the form $\mathbf{A} \ddot{\mathbf{x}}+\mathbf{E} \dot{\mathbf{x}}+\mathbf{L x}=\mathbf{F}$, where $\mathbf{A}, \mathbf{E}, \mathbf{L}$ and $\mathbf{F}$ denote, respectively, $m \times l$ matrices and an $l$ vector, Eq. (1) is recast into

$$
\overline{\mathbf{M}}_{\mathbf{x}} \ddot{\mathbf{x}}+\overline{\mathbf{C}}_{\mathbf{x}} \dot{\mathbf{x}}+\overline{\mathbf{K}}_{\mathbf{x}} \mathbf{x}+\overline{\mathbf{\Phi}}_{\mathbf{x}}(\mathbf{x}, \dot{\mathbf{x}}, \ddot{\mathbf{x}})=\overline{\mathbf{f}}_{d, \mathbf{x}}(t)+\overline{\mathbf{Q}}_{\mathbf{x}}(t) .
$$

In Eq. (2), $\overline{\mathbf{M}}_{\mathbf{x}}, \overline{\mathbf{C}}_{\mathbf{x}}$ and $\overline{\mathbf{K}}_{\mathbf{x}}$ denote the augmented $(l+m) \times l$ mass, damping and stiffness parameter matrices, which have the form [7]

$$
\overline{\mathbf{M}}_{\mathbf{x}}=\left[\begin{array}{c}
\left(\mathbf{I}_{l}-\mathbf{A}^{+} \mathbf{A}\right) \mathbf{M}_{\mathbf{x}} \\
\mathbf{A}
\end{array}\right], \quad \overline{\mathbf{C}}_{\mathbf{x}}=\left[\begin{array}{c}
\left(\mathbf{I}_{l}-\mathbf{A}^{+} \mathbf{A}\right) \mathbf{C}_{\mathbf{x}} \\
\mathbf{E}
\end{array}\right], \quad \overline{\mathbf{K}}_{\mathbf{x}}=\left[\begin{array}{c}
\left(\mathbf{I}_{l}-\mathbf{A}^{+} \mathbf{A}\right) \mathbf{K}_{\mathbf{x}} \\
\mathbf{L}
\end{array}\right],
$$

where $\mathbf{I}_{l}$ denotes the $l \times l$ identity matrix, and “+" represents the M-P matrix inverse operation. Further, the augmented $(l+m)$ vector of the system nonlinearities, and the corresponding periodic and stochastic $(l+m)$ vectors of the excitation are given by

$$
\overline{\mathbf{\Phi}}_{\mathbf{x}}=\left[\begin{array}{c}
\left(\mathbf{I}_{l}-\mathbf{A}^{+} \mathbf{A}\right) \boldsymbol{\Phi}_{\mathbf{x}} \\
\mathbf{0}
\end{array}\right], \overline{\mathbf{f}}_{d, \mathbf{x}}(t)=\left[\begin{array}{c}
\left(\mathbf{I}_{l}-\mathbf{A}^{+} \mathbf{A}\right) \mathbf{f}_{d, \mathbf{x}}(t) \\
\mathbf{F}
\end{array}\right], \overline{\mathbf{Q}}_{\mathbf{x}}(t)=\left[\begin{array}{c}
\left(\mathbf{I}_{l}-\mathbf{A}^{+} \mathbf{A}\right) \mathbf{Q}_{\mathbf{x}}(t) \\
\mathbf{F}
\end{array}\right]
$$


A detailed derivation of Eqs. (2)-(4) is found in [7, 12].

Next, considering that the MDOF system in Eq. (2) is subject to combined deterministic and stochastic loads, it is assumed that the corresponding response also consists of a periodic and a stochastic component, namely $\mathbf{x}(t)=\mathbf{x}_{s}(t)+\mathbf{x}_{d}(t)$. Further, assuming that the stochastic excitation is modeled as a zero-mean Gaussian process, ensemble averaging Eq. (2) yields

$$
\overline{\mathbf{M}}_{\mathbf{x}} \ddot{\mathbf{x}}_{d}+\overline{\mathbf{C}}_{\mathbf{x}} \dot{\mathbf{x}}_{d}+\overline{\mathbf{K}}_{\mathbf{x}} \mathbf{x}_{d}+\mathbb{E}\left[\overline{\mathbf{\Phi}}_{\mathbf{x}}\left(\mathbf{x}_{s}+\mathbf{x}_{d}, \dot{\mathbf{x}}_{s}+\dot{\mathbf{x}}_{d}, \ddot{\mathbf{x}}_{s}+\ddot{\mathbf{x}}_{d}\right)\right]=\overline{\mathbf{f}}_{d, \mathbf{x}}(t),
$$

which consists of a periodic and a stochastic response component too. In the ensuing analysis, first, the harmonic balance methodology is used in conjunction with the concept of the generalized inverse matrix for treating the deterministic component in Eq. (5). Then, the stochastic component of the system is treated by applying the generalized statistical linearization $[12,13]$.

\subsection{Application of the harmonic balance method for treating the periodic component of the response}

Assume that $\overline{\mathbf{f}}_{d, \mathbf{x}}(t)$ in Eq. (4) is modeled as a monofrequency function of period $T=\frac{2 \pi}{\omega_{d}}$. Then, $\overline{\mathbf{f}}_{d, \mathbf{x}}(t)=\overline{\mathbf{f}}_{d_{1}, \mathbf{x}} \cos \left(\omega_{d} t\right)+\overline{\mathbf{f}}_{d_{2}, \mathbf{x}} \sin \left(\omega_{d} t\right)$, where $\overline{\mathbf{f}}_{d_{1}, \mathbf{x}}$ and $\overline{\mathbf{f}}_{d_{2}, \mathbf{x}}$ are the constant coefficient $(l+m)$ vectors, and thus, the deterministic response of the system in Eq. (5) becomes $\mathbf{x}_{d}(t)=$ $\mathbf{x}_{d_{1}} \cos \left(\omega_{d} t\right)+\mathbf{x}_{d_{2}} \sin \left(\omega_{d} t\right)$, where $\mathbf{x}_{d_{1}}, \mathbf{x}_{d_{2}}$ are constant $l$ vectors. Next, applying the harmonic balance method yields the overdetermined system of equations

$$
\mathbf{P u}=\mathbf{v}
$$

where the $2(l+m) \times 2 l$ matrix $\mathbf{P}$, and the $2 l$ - and $2(l+m)$ vectors $\mathbf{u}, \mathbf{v}$ are given by

$$
\mathbf{P}=\left[\begin{array}{cc}
\overline{\mathbf{K}}_{\mathbf{x}}-\omega_{d}^{2} \overline{\mathbf{M}}_{\mathbf{x}} & \omega_{d} \overline{\mathbf{C}}_{\mathbf{x}} \\
-\omega_{d} \overline{\mathbf{C}}_{\mathbf{x}} & \overline{\mathbf{K}}_{\mathbf{x}}-\omega_{d}^{2} \overline{\mathbf{M}}_{\mathbf{x}}
\end{array}\right], \mathbf{u}=\left[\begin{array}{c}
\mathbf{x}_{d_{1}} \\
\mathbf{x}_{d_{2}}
\end{array}\right], \mathbf{v}=\left[\begin{array}{l}
\overline{\mathbf{f}}_{d_{1}}-\frac{2}{T} \int_{0}^{T} \mathbb{E}\left[\overline{\mathbf{\Phi}}_{\mathbf{x}}\right] \cos \left(\omega_{d} t\right) d t \\
\overline{\mathbf{f}}_{d_{2}}-\frac{2}{T} \int_{0}^{T} \mathbb{E}\left[\overline{\mathbf{\Phi}}_{\mathbf{x}}\right] \sin \left(\omega_{d} t\right) d t
\end{array}\right]
$$

The solution of the system in Eq. (6) leads to the computation of the deterministic component of the response. In this regard, utilizing the generalized matrix inverses theory implies [14]

$$
\mathbf{u}=\mathbf{P}^{+} \mathbf{v}+\left(\mathbf{I}-\mathbf{P}^{+} \mathbf{P}\right) \mathbf{y}
$$

which, in essence, corresponds to a family of solutions, since $\mathbf{y}$ is an arbitrary $2 l$ vector. However, the matrix $\overline{\mathbf{M}}_{\mathbf{x}}$ in the diagonal entries of matrix $\mathbf{P}$ in Eq. (7) ensures that the columns of the latter are independent of each other or, equivalently, that matrix $\mathbf{P}$ has full column rank. Therefore, it holds that $\mathbf{P}^{+}=\left(\mathbf{P}^{*} \mathbf{P}\right)^{-1} \mathbf{P}^{*}$, and thus, Eq. (8) attains a unique solution corresponding to the deterministic response component of the system in Eq. (2).

\subsection{Application of the statistical linearization method for treating the stochastic compo- nent of the response}

The stochastic component $\mathbf{x}_{s}(t)$ of the system response is treated by resorting to the generalized statistical linearization methodology for systems with singular parameter matrices [12, 13]; see also $[16,17,18,19,20,21,22,23]$ for a broader perspective, as well as several examples pertaining to application of the method. First, the difference between the systems in Eqs. (2) and (5) is formed,

$$
\overline{\mathbf{M}}_{\mathbf{x}} \ddot{\mathbf{x}}_{s}+\overline{\mathbf{C}}_{\mathbf{x}} \dot{\mathbf{x}}_{s}+\overline{\mathbf{K}}_{\mathbf{x}} \mathbf{x}_{s}+\tilde{\boldsymbol{\Phi}}_{\mathbf{x}}\left(\mathbf{x}_{s}, \mathbf{x}_{d}\right)=\overline{\mathbf{Q}}_{\mathbf{x}}(t),
$$


where $\tilde{\mathbf{\Phi}}_{\mathbf{x}}\left(\mathbf{x}_{s}, \mathbf{x}_{d}\right)=\overline{\mathbf{\Phi}}_{\mathbf{x}}\left(\mathbf{x}_{s}+\mathbf{x}_{d}, \dot{\mathbf{x}}_{s}+\dot{\mathbf{x}}_{d}, \ddot{\mathbf{x}}_{s}+\ddot{\mathbf{x}}_{d}\right)-\mathbb{E}\left[\overline{\mathbf{\Phi}}_{\mathbf{x}}\left(\mathbf{x}_{s}+\mathbf{x}_{d}, \dot{\mathbf{x}}_{s}+\dot{\mathbf{x}}_{d}, \ddot{\mathbf{x}}_{s}+\ddot{\mathbf{x}}_{d}\right)\right]$ is the zero-mean vector of the system nonlinearities. Then, the linear equivalent system to Eq. (9) is written as $[12,16]$

$$
\left(\overline{\mathbf{M}}_{\mathbf{x}}+\overline{\mathbf{M}}_{e}\right) \ddot{\mathbf{x}}_{s}+\left(\overline{\mathbf{C}}_{\mathbf{x}}+\overline{\mathbf{C}}_{e}\right) \dot{\mathbf{x}}_{s}+\left(\overline{\mathbf{K}}_{\mathbf{x}}+\overline{\mathbf{K}}_{e}\right) \mathbf{x}_{s}=\overline{\mathbf{Q}}_{\mathbf{x}}(t) .
$$

Subsequently, the error function, which is defined as the difference between Eqs. (9) and (10), is formed and minimized by adopting the mean square minimization criterion $[12,13,16]$. In this regard, the elements of the $(l+m) \times l$ matrices $\overline{\mathbf{M}}_{e}, \overline{\mathbf{C}}_{e}$ and $\overline{\mathbf{K}}_{e}$ are given by pertinent closed form expressions; more details are found in [13].

Since the nonlinear vector $\tilde{\Phi}_{\mathbf{x}}\left(\mathbf{x}_{s}, \mathbf{x}_{d}\right)$ depends on the deterministic and stochastic components of the response, the elements $m_{i j}^{e}, c_{i j}^{e}, k_{i j}^{e}$ of matrices $\overline{\mathbf{M}}_{e}, \overline{\mathbf{C}}_{e}$ and $\overline{\mathbf{K}}_{e}$ are also timedependent. Nevertheless, the slowly varying over a period $T$ of oscillation components of $\overline{\mathbf{M}}_{e}, \overline{\mathbf{C}}_{e}$ and $\overline{\mathbf{K}}_{e}$ are approximated by their average over $T[15,24]$, i.e., $\overline{\mathbf{M}}_{e} \approx \overline{\mathbf{M}}_{e}^{a v}, \overline{\mathbf{C}}_{e} \approx \overline{\mathbf{C}}_{e}^{a v}$ and $\overline{\mathbf{K}}_{e} \approx \overline{\mathbf{K}}_{e}^{a v}$; indicatively, $\overline{\mathbf{M}}_{e}^{a v}=\frac{1}{T} \int_{0}^{T} \overline{\mathbf{M}}_{e} d t$. This provides with an approximation to the system in Eq. (10), given by the equivalent system

$$
\left(\overline{\mathbf{M}}_{\mathbf{x}}+\overline{\mathbf{M}}_{e}^{a v}\right) \ddot{\mathbf{x}}_{s}+\left(\overline{\mathbf{C}}_{\mathbf{x}}+\overline{\mathbf{C}}_{e}^{a v}\right) \dot{\mathbf{x}}_{s}+\left(\overline{\mathbf{K}}_{\mathbf{x}}+\overline{\mathbf{K}}_{e}^{a v}\right) \mathbf{x}_{s}=\overline{\mathbf{Q}}_{\mathbf{x}}(t) .
$$

Further, the response statistics of the equivalent system in Eq. (11) are determined by employing a frequency domain approach. In this regard, the frequency response function matrix of the system in Eq. (11) is derived and used in conjunction with the generalized input-output relationship for systems with singular parameter matrices, i.e., $\mathbf{S}_{\mathbf{x}}(\omega)=\boldsymbol{\alpha}_{\mathbf{x}}(\omega) \mathbf{S}_{\overline{\mathbf{Q}}_{\mathbf{x}}}(\omega) \boldsymbol{\alpha}_{\mathbf{x}}^{\mathrm{T} *}(\omega)$, for determining the response power spectrum $\mathbf{S}_{\mathbf{x}_{s}}(\omega)$ [13] Finally, the second order response statistics of the equivalent system in Eq. (11) are computed by [16]

$$
\mathbb{E}\left[\mathbf{x}_{s}^{2}(i)\right]=\int_{-\infty}^{\infty} S_{\mathbf{x}_{s}(i) \mathbf{x}_{s}(i)}(\omega) d \omega, i=1,2, \ldots, l .
$$

Eq. (12) in conjunction with the generalized input-output relationship for systems with singular parameter matrices, as well as Eq. (8), constitute a coupled nonlinear system of equations to be solved for determining the system response. For its solution, the following iterative procedure is used: $i$. The nonlinear vector $\tilde{\Phi}_{\mathbf{x}}\left(\mathbf{x}_{s}, \mathbf{x}_{d}\right)$ in the governing equations of motion is set equal to the null vector and the deterministic response $\mathbf{x}_{d}$ is obtained. $i i$. The variance of the stochastic response $\mathbf{x}_{s}$ is derived by using the generalized input-output relationship in conjunction with Eq. (12). iii. Using step (ii.), Eq. (8) leads to the derivation of the deterministic response, which, in turn, results in calculating the values $\overline{\mathbf{M}}_{e}^{a v}, \overline{\mathbf{C}}_{e}^{a v}$ and $\overline{\mathbf{K}}_{e}^{a v}$. iv. Steps (ii.) and (iii.) are repeated until reaching satisfactory accuracy for the response variance.

\section{NUMERICAL EXAMPLE}

In this example, the 2-DOF system in Fig. 1 is considered, where mass $m_{1}$ is connected to the foundation by a nonlinear damper and a nonlinear spring of forces $c_{1} \dot{q}_{1}\left(1+\varepsilon_{1} \dot{q}_{1}^{2}\right)$ and $k_{1} q_{1}\left(1+\varepsilon_{2} q_{1}^{2}\right)$, respectively. Further, $q_{i}$ denotes the displacement of the $i$-th mass, and $\varepsilon_{1}, \varepsilon_{2}$ the magnitude of the nonlinearities. In addition, mass $m_{2}$ is connected to $m_{1}$ by a linear spring of stiffness $k_{2}$ and a linear damper of damping coefficient $c_{2}$. The system is excited by a combination of a stochastic force $Q_{1}(t)$, which is modeled as a Gaussian white noise stochastic process with spectral density $S_{0}$, and a deterministic force of the form $f_{d_{2}, 1} \sin \left(\omega_{d} t\right)$. The following set of parameter values is considered: $m_{1}=m_{2}=1, c_{1}=c_{2}=0.2, k_{1}=k_{2}=1, S_{0}=10^{-2}$ $(0<\omega<2 \pi), f_{d_{2}, 1}=0.4, \omega_{d}=\pi$ and $\varepsilon_{1}=\varepsilon_{2}=10$. 


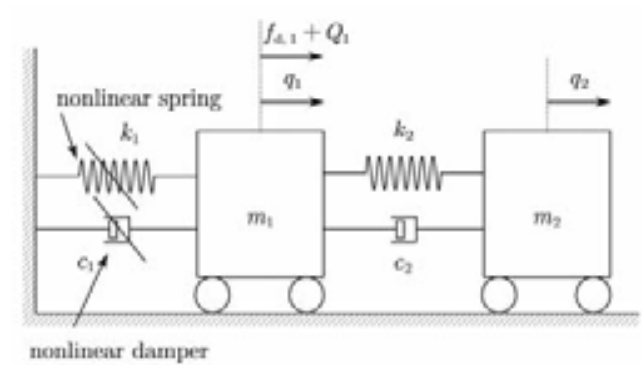

Figure 1: A 2-DOF nonlinear system subject to periodic and stochastic excitations.

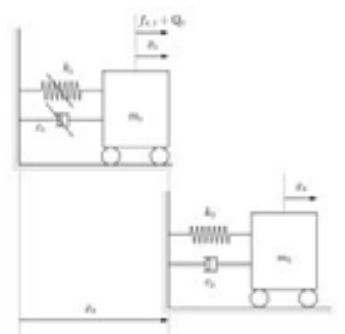

Figure 2: The nonlinear system of Fig. 1 modeled by employing an additional redundant coordinate.

Next, considering the redundant coordinates vector $\mathbf{x}^{\mathrm{T}}=\left[\begin{array}{lll}x_{1} & x_{2} & x_{3}\end{array}\right]$, the 2-DOF system of Fig. 1 is decomposed into its partial subsystems, as shown in Fig. 2. The system constraints equation is formed (see section 2), where $\mathbf{A}=\left[\begin{array}{lll}1 & -1 & 0\end{array}\right], \mathbf{E}=\mathbf{L}=\mathbf{0}_{1 \times 3}$ and $F=0$. Thus, the parameter matrices defined in Eq. (3) are determined, and the $(l+m)$ vectors in Eq. (4) are computed. For the application of the harmonic balance method, the system response is decomposed into its stochastic $\mathbf{x}_{s}$ and deterministic $\mathbf{x}_{d}$ components, and ensemble averaging the expression for $\bar{\Phi}_{\mathrm{x}}$ yields

$$
\left(\mathbb{E}\left[\overline{\mathbf{\Phi}}_{\mathbf{x}}\right]\right)^{\mathrm{T}}=\left(c_{1} \varepsilon_{1}\left(\dot{x}_{d, 1}^{3}+3 \dot{x}_{d, 1} \sigma_{\dot{x}_{s, 1}}^{2}\right)+k_{1} \varepsilon_{2}\left(x_{d, 1}^{3}+3 x_{d, 1} \sigma_{x_{s, 1}}^{2}\right)\right)\left[\begin{array}{llll}
0.5 & 0.5 & 0 & 0
\end{array}\right] .
$$

Then, taking into account that the $8 \times 6$ matrix $\mathbf{P}$ has full rank (see Eq. (7)), the overdetermined system in Eq. (6) leads to a uniquely defined periodic response component. Next, the iterative scheme in section 2.2 is employed to solve the coupled set of algebraic equations formed by Eqs. (12) and Eq. (8), and thus, to derive the variance of the stochastic response. The scheme is initialized by using $\overline{\mathbf{C}}_{e}^{a v}=\mathbf{0}, \overline{\mathbf{K}}_{e}^{a v}=\mathbf{0}$ and $x_{d_{1}}=x_{d_{2}}=0$. The stochastic and deterministic components are derived based on the criterion $\left|\frac{\overline{\mathbf{C}}_{e, j+1}^{a v}-\overline{\mathbf{C}}_{e, j}^{a v}}{\overline{\mathbf{C}}_{e, j}}\right|<10^{-5}$ and $\left|\frac{\overline{\mathbf{K}}_{e, j+1}^{a v}-\overline{\mathbf{K}}_{e, j}^{a v}}{\overline{\mathbf{K}}_{e, j}^{a v}}\right|<10^{-5}$, as well as a similar criterion for $x_{d_{1}}, x_{d_{2}}$. The iterative scheme is applied until reaching satisfactory accuracy for the response displacement and velocity variances. Finally, considering that $\mathbf{x}(t)=\mathbf{x}_{s}(t)+\mathbf{x}_{d}(t)$ and successively ensemble and temporal averaging the expression $\mathbf{x}_{d}(t)=\mathbf{x}_{d_{1}} \cos \left(\omega_{d} t\right)+\mathbf{x}_{d_{2}} \sin \left(\omega_{d} t\right)$ implies

$$
\left\langle\mathbb{E}\left[x_{i}^{2}\right]\right\rangle=\sigma_{x_{s, 1}}^{2}+\frac{x_{d_{1}, i}^{2}+x_{d_{2}, i}^{2}}{2},\left\langle\mathbb{E}\left[\dot{x}_{i}^{2}\right]\right\rangle=\sigma_{\dot{x}_{s, 1}}^{2}+\frac{\omega_{d}^{2}\left(x_{d_{1}, i}^{2}+x_{d_{2}, i}^{2}\right)}{2}, i=1,2,3,
$$

where $\langle\cdot\rangle$ denotes the temporal averaging operation.

Eq. (14), in conjunction with the results of the iterative scheme above yield $\sigma_{x_{1}}^{2}=0.0705$, $\sigma_{\dot{x}_{1}}^{2}=0.0704$ and $\sigma_{x_{3}}^{2}=0.0617, \sigma_{\dot{x}_{3}}^{2}=0.1098$. Finally, applying the standard solution framework proposed in [15] for deriving the system response variance yields $\sigma_{q_{1}}^{2}=0.0705$, $\sigma_{\dot{q}_{1}}^{2}=0.0704$ and $\sigma_{q_{2}}^{2}=0.0617, \sigma_{\dot{q}_{2}}^{2}=0.1098$. Clearly, comparing the results above, it is seen that the herein proposed framework is in total agreement with the standard approach in [15].

\section{CONCLUSIONS}

In this paper, the response of multi-degree-of-freedom nonlinear systems with singular parameter matrices subject to combined stochastic and deterministic excitations has been determined by resorting to a generalized inverse matrix-based approach. Singular matrices appeared due to a redundant coordinates formulation of the system governing equations of motion. The 
latter is associated with solution frameworks of reduced computational cost for certain classes of complex multi-body systems and/or systems with constraints. In this context, considering that the system excitation consists of a periodic and a stochastic component, the corresponding response has been decomposed in two parts, namely a periodic and a stochastic. Then, the system response has been determined by utilizing the generalized matrix inverses theory, in conjunction with the harmonic balance method and the generalized statistical linearization method for systems with singular parameter matrices. The validity of the proposed technique has been demonstrated by pertinent numerical examples.

\section{ACKNOWLEDGMENTS}

The research work herein was supported by the Hellenic Foundation for Research and Innovation under Grant/Project No. 1261. This support is gratefully acknowledged.

\section{REFERENCES}

[1] A.D. Karageorgos, L. Moysis, V.C. Fragkoulis, I.A. Kougioumtzoglou, A.A. Pantelous, Random vibration of linear systems with singular matrices based on Kronecker canonical forms of matrix pencils. Mech. Syst. Sig. Process., 161, 107896, 2021.

[2] A. Pirrotta, I.A. Kougioumtzoglou, A. Di Matteo, V.C. Fragkoulis, A.A. Pantelous, C. Adam, Deterministic and random vibration of linear systems with singular parameter matrices and fractional derivative terms. J. Eng. Mech., 147(6), 04021031, 2021.

[3] F.E. Udwadia, R.E. Kalaba, A new perspective on constrained motion. Proc. R. Soc. London, Ser. A: Math. Phys. Sci., 439(1906), 407-410, 1992.

[4] A. Schutte, F.E. Udwadia, New approach to the modeling of complex multibody dynamical systems. J. Appl. Mech., 78(2), 021018-1-021018-11, 2011.

[5] C.M. Pappalardo, D. Guida, On the Lagrange multipliers of the intrinsic constraint equations of rigid multibody mechanical systems. Arch. Appl. Mech., 88(3), 419-451, 2018.

[6] V.C. Fragkoulis, I.A. Kougioumtzoglou, A.A. Pantelous, A. Pirrotta, Higher order matrix differential equations with singular coefficient matrices. AIP Conf. Proc., 1648(1), 340002, 2015.

[7] V.C. Fragkoulis, I.A. Kougioumtzoglou, A.A. Pantelous, Linear random vibration of structural systems with singular matrices. J. Eng. Mech., 142(2), 04015081, 2016 a.

[8] A.A. Pantelous, A. Pirrotta, Modal analysis of multi-degrees-of-freedom systems with singular matrices: Analytical dynamics approach. J. Eng. Mech., 143(6), 06017005, 2017.

[9] A. Pirrotta, I.A. Kougioumtzoglou, A.A. Pantelous, Stochastic response determination of structural systems modeled via dependent coordinates: A frequency domain treatment based on generalized modal analysis. Meccanica, 54(9), 1421-1431, 2019.

[10] G.D. Pasparakis, V.C. Fragkoulis, M. Beer, Harmonic wavelets based response evolutionary power spectrum determination of linear and nonlinear structural systems with singular matrices. Mech. Syst. Sig. Process., 149, 107203, 2021. 
[11] V.C. Fragkoulis, I.A. Kougioumtzoglou, A.A. Pantelous, M. Beer, Joint statistics of natural frequencies corresponding to structural systems with singular random parameter matrices. J. Eng. Mech. (Forthcoming).

[12] V.C. Fragkoulis, I.A. Kougioumtzoglou, A.A. Pantelous, Statistical linearization of nonlinear structural systems with singular matrices. J. Eng. Mech., 142(9), 04016063, 2016b.

[13] I.A. Kougioumtzoglou, V.C. Fragkoulis, A.A. Pantelous, A. Pirrotta, Random vibration of linear and nonlinear structural systems with singular matrices: A frequency domain approach. J. Sound Vib., 404, 84-101, 2017.

[14] S.L. Campbell, C.D. Meyer, Generalized inverses of linear transformations. SIAM, 2009.

[15] P.D. Spanos, Y. Zhang, F. Kong, Formulation of statistical linearization for M-D-O-F systems subject to combined periodic and stochastic excitations. J Appl Mech, 86(10), $101003,2019$.

[16] J.B. Roberts, P.D. Spanos, Random vibration and statistical linearization. Courier, 2003.

[17] V.C. Fragkoulis, I.A. Kougioumtzoglou, A.A. Pantelous, M. Beer, Non-stationary response statistics of nonlinear oscillators with fractional derivative elements under evolutionary stochastic excitation. Nonlinear Dyn., 97(4), 2291-2303, 2019.

[18] I.P. Mitseas, M. Beer, Modal decomposition method for response spectrum based analysis of nonlinear and non-classically damped systems. Mech. Syst. Sig. Process., 131, 469-485, 2019.

[19] M.G.R. Faes, V.C. Fragkoulis, P. Ni, D.J. Jerez, M.A. Valdebenito, M. Beer, Operator Norm-based Statistical Linearization to Bound the First Excursion Probability of Nonlinear Structures Subjected to Imprecise Stochastic Loading. ASCE-ASME J. Risk Uncertainty Eng. Syst., Part A: Civ. Eng. (Forthcoming).

[20] I.P. Mitseas, I.A. Kougioumtzoglou, A. Giaralis, M. Beer, A novel stochastic linearization framework for seismic demand estimation of hysteretic MDOF systems subject to linear response spectra. Structural Safety, 72, 84-98, 2018.

[21] I.P. Mitseas, I.A. Kougioumtzoglou, P.D. Spanos, M. Beer, Nonlinear MDOF structural system survival probability determination subject to evolutionary stochastic excitation, $J$. Mech. Eng., 62(7-8), 440-451, 2016.

[22] I.P. Mitseas, M. Beer, Fragility analysis of nonproportionally damped inelastic MDOF structural systems exposed to stochastic seismic excitation, Computers and Structures, 226, doi: 10.1016/j.compstruc.2019.106129, 2020.

[23] I.P Mitseas, M. Beer M., First-excursion stochastic incremental dynamics methodology for hysteretic structural systems subject to seismic excitation, Computers and Structures, 242, doi: 10.1016/j.compstruc.2020.106359, 2021.

[24] P. Ni, V.C. Fragkoulis, F. Kong, I.P. Mitseas, M. Beer, Response determination of nonlinear systems with singular matrices subject to combined stochastic and deterministic excitations. ASCE-ASME J. Risk Uncertainty Eng. Syst., Part A: Civ. Eng., 7(4), 04021049, 2021. 